\title{
Design of a wearable interface for lightweight robotic arm for people with mobility impairments
}

This is the peer reviewed version of the following article:

Original:

LISINI BALDI, T., Spagnoletti, G., M., D., Prattichizzo, D. (2017). Design of a wearable interface for lightweight robotic arm for people with mobility impairments. In Proc. 2017 International Conference on Rehabilitation Robotics (ICORR) (pp.1567-1573). New York : IEEE [10.1109/ICORR.2017.8009471].

Availability:

This version is availablehttp://hdl.handle.net/11365/1012989

since 2019-05-07T17:08:30Z

Publisher:

IEEE

Published:

DOI:10.1109/ICORR.2017.8009471

Terms of use:

Open Access

The terms and conditions for the reuse of this version of the manuscript are specified in the publishing policy. Works made available under a Creative Commons license can be used according to the terms and conditions of said license.

For all terms of use and more information see the publisher's website.

(Article begins on next page) 


\title{
Design of a Wearable Interface for Lightweight Robotic Arm for People with Mobility Impairments
}

\author{
Tommaso Lisini Baldi ${ }^{1,2}$, Giovanni Spagnoletti ${ }^{1}$, Mihai Dragusanu ${ }^{1}$, and Domenico Prattichizzo ${ }^{1,2}$
}

\begin{abstract}
Many common activities of daily living like open a door or fill a glass of water, which most of us take for granted, could be an insuperable problem for people who have limited mobility or impairments. For years the unique alternative to overcame this limitation was asking for human help. Nowadays thanks to recent studies and technology developments, having an assistive devices to compensate the loss of mobility is becoming a real opportunity. Off-the-shelf assistive robotic manipulators have the capability to improve the life of people with motor impairments. Robotic lightweight arms represent one of the most spread solution, in particular some of them are designed specifically to be mounted on wheelchairs to assist users in performing manipulation tasks. On the other hand, usually their control interface relies on joystick and buttons, making the use very challenging for people affected by impaired motor abilities. In this paper, we present a novel wearable control interface for users with limb mobility impairments. We make use of muscles residual motion capabilities, captured through a Body-Machine Interface based on a combination of head tilt estimation and electromyography signals. The proposed BMI is completely wearable, wireless and does not require frequently long calibrations. Preliminary experiments showed the effectiveness of the proposed system for subjects with motor impairments, allowing them to easily control a robotic arm for activities of daily living.
\end{abstract}

Index Terms-Wheelchair-mounted robotic arm, Head tracking, EMG signals, Inertial and magnetic sensors, Wearable technology, Assistive technology.

\section{INTRODUCTION}

According to the European Health and Social Integration Survey over 49 million people need assistance in their daily lives [1]. Assistive technologies like powered wheelchairs, walkers, canes, and prosthetic devices have greatly enhanced the quality of life for individuals with disabilities. Nevertheless, people with limited limbs usage have difficulty in performing Activities of Daily Living (ADLs) such as picking up and objects, opening doors, filling a glass of water, etc. Interest and effort in this field have led to design Wheelchair-Mounted Robotic Manipulators (WMRMs) to increase autonomy in manipulating objects in ADL for people with upper extremity reduced mobility, like persons with spinal cord injuries [2], [3]. Several robotic arms designed as WMRM are commercially available. The Manus

The research leading to these results has received funding from European Union's Horizon 2020 Research and Innovation Programme under Grant Agreement n. 688857 of the project SoftPro

1 Authors are with the Department of Information Engineering and Mathematics, University of Siena, 53100 Siena, Italy. \{lisini, spagnoletti, prattichizzo\}@diism.unisi.it

2 Authors are with the Department of Advanced Robotics, Istituto Italiano di Tecnologia, Genova, 16163, Italy. \{tommaso.lisini, domenico.prattichizzo\}eit.it manipulator, produced by Exact Dynamics, is a 6 Degrees of Freedom (DoFs) robot that can be mounted on a wheelchair system [4]. Kinova developed JACO and MICO lightweight robots ready to be carried on a wheelchair to help people with limb impairments in their ADL [5]. A common drawback for this robot family is having more DoF than the dimensionality of their control interface, thus resulting a hard usability for impaired users.

Recent works studied solutions for making such robots fully or partially autonomous [6], [7]. However, it has been proven that patients prefer to retain as much control as possible when working with assistive devices [3]. Joysticks and gamepads are the most widespread input technologies, thanks to their quick integration and easiness of use, but they have to be operated by hand. Very often people with upper limbs impairments cannot move their hands and arms with fine and precise gestures. Existing Human-Computer Interaction (HCI) modalities [3] as well as emerging Brain Computer Interfaces [8] and state-of-the-art computer vision systems [9] have been shown to be suitable controllers for WMRM systems. Prior investigations in HCI for people with upper extremity impaired or quadriplegics has resulted in several Body-Machine Interfaces (BMI) as speech and gesture recognition [10], [11], tongue movements [12], Electrooculography [13], and Electromyography (EMG) [14]. Voice control interface is a common solution, but the reliability of the speech recognition decrease with increasing of the environmental noise, so it becomes unreliable in crowded spaces, or noisy outdoor scenarios. An alternative control method relies on gesture motion recognition and body tracking. Concerning this, several techniques have been developed such as optical trackers, exoskeletons, camera tracking algorithms, and fabric-integrated sensors. Accurate optical tracking systems such as Vicon (Vicon Motion Systems, UK) and Optitrack (NaturalPoint Inc., USA) exploit active or passive optical markers to estimate human body configuration with high precision and accuracy. The main drawback of such approaches is the need of a high-cost structured environment. Exoskeletons allow to accurately estimate the human pose thanks to their rigid structure and high quality sensors [7]. Disadvantages result in cost, weight, and poor portability. Camera-based tracking algorithms became a widespread solution due to improvements in computer vision techniques and progressive growth in GPU computational capabilities [15], [16]. However these techniques have some limitations: RGB-D cameras might not work properly in an outdoor environment due to the infrared interference, and occlusions may induce a poor estimation. A promising way 

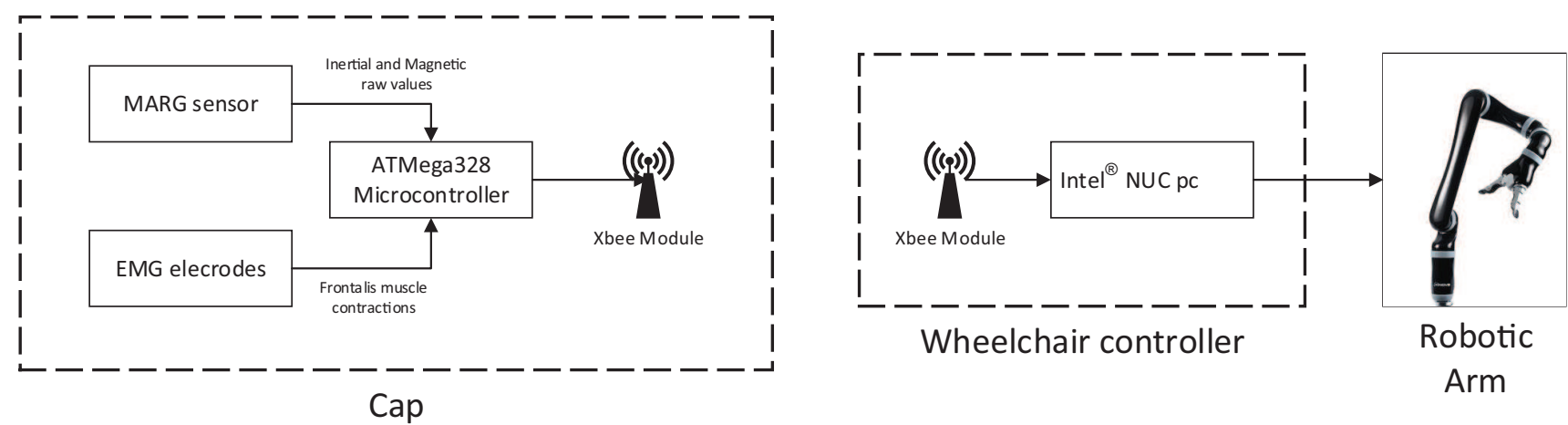

Fig. 1. System architecture scheme. A cap instrumented with a MARG board and three EMG electrodes is used to record and wireless transmit to a PC both the detected frontalis muscle contractions and the inertial and magnetic values. Two Xbee Series 1 boards create a wireless serial link between the cap and the wheelchair controller. The controller is based on an Intel ${ }^{\circledR}$ NUC pc in which are executed at the same time both the minimization algorithm and the robot control main program. The Intel ${ }^{\circledR}$ NUC pc and the Kinova arm can be mounted on a powered wheelchair.

to track the user and control a robotic manipulator is based on Micro Electro-Mechanical Systems (MEMS) technology. In particular, a MARG (Magnetic, Angular Rate, and Gravity) board consists of a MEMS triaxial gyroscope, accelerometer, and magnetometer. The board can be easily integrated with a wearable device and used to command the motion of a robotic aid. Tracking systems based on this technology are commercially available and allow to accurately track, both in outdoor and indoor environments, under different light conditions and free from grounded hardware [17]-[19].

In this paper, we present a novel Body Machine Interface to control an assistive robotic arm. The proposed BMI extracts signals from body motions exploiting residual movements available even in people with severe impairments. Our system employs a MARG sensor for estimating the patient's head orientation and EMG electrodes for detecting muscle contractions. The patient can thus drive the assistive robot tilting the head and contracting the frontalis muscles. This choice fitted the requirements of designing a low cost interface capable of working in unstructured environments with varying light conditions, being portable and independent from grounded tracking hardware. Moreover, since the goal is to create a wearable system, an instrumented cap represents a good deal between user customization, portability, and tracking capabilities. Pilot experimental results show the effectiveness of the proposed approach, allowing the patient to grasp a bottle and fill a glass of water in about a minute and half.

The paper is organized as follows. Section II presents the wearable interface for controlling a lightweight robotic arm using EMG signals and head motion. Section III details the head tilt estimation algorithm. Section IV reports the EMG signals acquisition and filtering procedure. In Section V we describe the case study. Finally, in Section VI conclusions are drawn and possible subjects of future research are outlined.

\section{WEARABLE INTERFACE}

A patient oriented control interface should be easy to use and effective. Based on this principle we build a system in which the user is both in control and is assisted by the
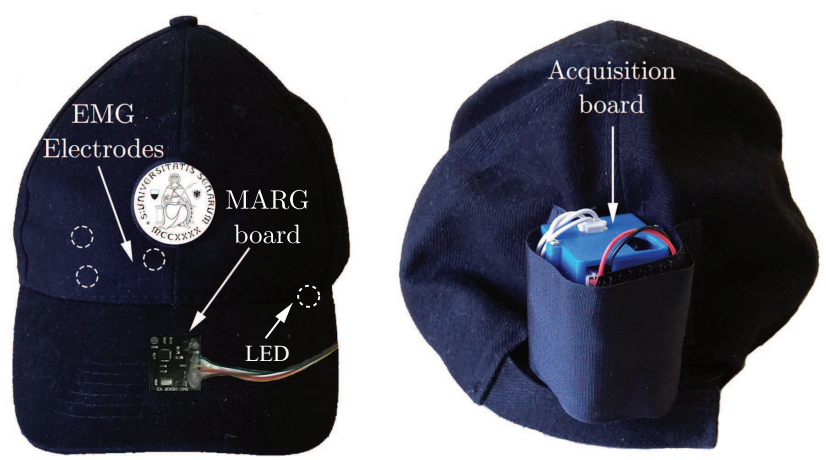

Fig. 2. The wearable control interface is made by a MARG board for tilt estimation, three EMG electrodes, and an acquisition board, based on a ATMega328 microcontroller. The system is capable of collecting the values from the MARG board and from the electrodes and send them wireless to the robot controller. A LED is used for provide the user with visual feedback

robot during the manipulation tasks. The wearable interface presented in this work aims at replacing dedicated inputs to fully control a robotic arm and operate the end effector. Usually joystick and buttons are used to control assistive robots, but they are not suitable for patients with disabilities, or upper limbs impairments. To overcame this functional limitation we replaced the buttons with the frontalis muscle contraction, and the joystick with the head inclination.

A cap (see Fig. 2), instrumented with a single MARG board and three electrodes (one channel bipolar EMG), is employed and used both as inputs for the tilt estimation and for the control mode selection. With the proposed system the user can switch between different robot control modes contracting the frontalis muscle, and move the gripper tilting the head. In Section III we report the algorithm for the tilt estimation, whereas in Section IV the EMG signal detections is fully detailed. An ATMega328 microcontroller, included in the cap, is in charge of collecting the values from the MARG board and from the EMG electrodes and send them through two Xbee ${ }^{\circledR}$ modules to the wheelchair controller. The acquisition rate of the inertial and magnetic values is 
$100 \mathrm{~Hz}$. A Kinova MICO2 robotic arm is actuated using an Intel ${ }^{\circledR}$ NUC PC under $C$ environment. The PC can be powered using the wheelchair battery and it is configured for a low power consumption. Thanks to these features the MICO2 arm could be mounted to the seat frame of a motorized wheelchair together with the controller. The architecture of the proposed wearable interface is illustrated in Fig. 1.

The proposed system required an initialization procedure composed of two steps. In the first phase, the user is asked to keep the head still for 3 seconds, time in which the MARG board collects 300 samples to calibrate the sensors, after that the current orientation is set as the initial one (see Section III for further details). In the second step, according to the maximum voluntary contraction (MVC) technique [20], the user is asked activate two times the frontalis muscle for three seconds to adjust levels and thresholds for a correct detection. A LED informs the user once the procedure is successfully completed. Thenceforth the user can start to operate the robot, switching between four different control modes selectable with the muscle contractions as detailed in Table I. The selection is fed back to the operator with the LED, that flashes $n$ times per second indicating the selected control mode (e.g., 3 times per second for the wrist rotation modality). Once the user has selected the desired control, he/she is able to move the robot tilting the head. Cap inclination and selected mode are transformed in robot motions in accordance with the following equation

$$
v_{k}= \begin{cases}\alpha_{k} \Psi_{i}, & \text { if }\left|\Psi_{i}\right|>\delta_{k} \\ 0, & \text { if }\left|\Psi_{i}\right| \leq \delta_{k}\end{cases}
$$

where $\Psi_{i}$ is the angle estimated using the algorithm detailed in Section III, $i=\{$ Roll, Pitch $\}$ is the current axis, $\delta_{k}$ represents a threshold to avoid undesired motion due to the head tremor and $\alpha_{k}$ is a parameter that converts the estimated angle into a velocity reference. The resulting speed $v_{k}$, along the $k$-th axis depends on the selected modality $k=$ $\{x, y, z, \theta, \phi, \psi\}$. Being $x-, y-, z-$ the axis w.r.t the robot base reference frame, and $\theta, \phi, \psi$ the robot wrist rotational axes. We discarded the Yaw angle, since it is not suitable for people with impairments. Moreover after some preliminary tests, we decide to use four different control modality instead of three to improve the usability and system effortlessness. We experimentally observed that keeping separated end effector translations and wrist rotations increases the learning effect and the familiarization with the system, thus reduce the uncertainty in the user's movement and consequently the motion control errors. We defined a high level control strategy based on a Finite State Machine (see Fig. 3) so that the user can switch between different control modalities (see Table I) thanks to the frontalis muscle contractions. Furthermore for the open and close fingers operation we decided to use only the EMG signals to control the grasp, since we can not control the speed of the fingers actuators. The event $e_{3}$ associated to the fingers motions produces a change in the end-effector state, i.e, from opened to close position and vice-versa, keeping the same control modality.

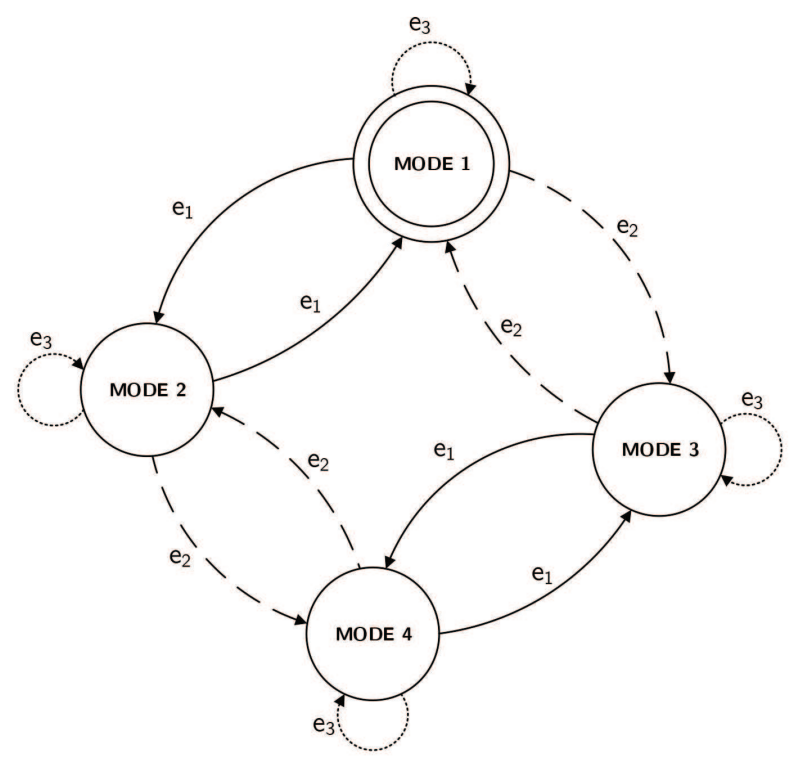

Fig. 3. The proposed finite state machine for the motion control of the robotic manipulator. $M O D E 1$ is the starting state. Events $e_{1}, e_{2}$ and $e_{3}$ are generated by contracting the frontalis muscle, that correspond to one, two, and three frontalis muscle contraction, respectively.

TABLE I

MODES DESCRIPTION

CAP TILT

\begin{tabular}{|c|c|c|}
\cline { 2 - 3 } \multicolumn{1}{c|}{} & Roll & Pitch \\
\hline Mode 1 & $\begin{array}{c}\text { Translation } \\
\text { Left/Right }\end{array}$ & $\begin{array}{c}\text { Translation } \\
\text { Forward/Backward }\end{array}$ \\
\hline Mode 2 & & $\begin{array}{c}\text { Translation } \\
\text { Up/Down }\end{array}$ \\
\hline Mode 3 & $\begin{array}{c}\text { Wrist rotation } \\
\text { clockwise/counter-clockwise }\end{array}$ & $\begin{array}{c}\text { Wrist } \\
\text { Lateral orientation }\end{array}$ \\
\hline Mode 4 & & $\begin{array}{c}\text { Wrist } \\
\text { Vertical orientation }\end{array}$ \\
\hline
\end{tabular}

\section{TILT ESTIMATION OF MARG SENSOR}

In the proposed work, we tested and implemented a GaussNewton method combined with a complementary filter [21] for the cap tilt estimation. As suggested in [19] this algorithm represents a good compromise since it achieved low estimation error, and it has only one parameter to be set. In this section, we briefly review the data fusion method and the procedure used to estimate the orientation of a single MARG with respect to an arbitrary position. The proposed algorithm uses quaternions to describe rotations. This redundant representation allow us to overcame the problem of rotating from a reference frame to another one without loss of precision due to the trigonometric functions.

At each time $t$, the gyroscope records the angular rates ${ }^{S} \omega_{x}(t),{ }^{S} \omega_{y}(t)$ and ${ }^{S} \omega_{z}(t)$ with respect to the $x-, y-$ and $z$-axis of the sensors board frame $S$. We can represent these values in a quaternion form

$$
{ }^{S} \boldsymbol{\omega}(t)=0+\boldsymbol{i}^{S} \omega_{x}(t)+\boldsymbol{j}^{S} \omega_{y}(t)+\boldsymbol{k}^{S} \omega_{z}(t)
$$


assuming that the first component of ${ }^{\boldsymbol{\omega}} \boldsymbol{\omega}(t)$ is the real number. We consider the sensor orientation rate as an infinitesimal quaternion variation,

$$
{ }^{S} \dot{g}(t)=\frac{1}{2}\left({ }^{S} \mathbf{q}(t-\delta t) \otimes{ }^{S} \boldsymbol{\omega}(t)\right)
$$

where ${ }^{S} \mathbf{q}(t-\delta t)$ is the latest estimated quaternion, ${ }^{S} \boldsymbol{\omega}(t)=\left[0{ }^{S} \omega_{x}(t){ }^{S} \omega_{y}(t){ }^{S} \omega_{z}(t)\right]^{T}$ is the angular rate vector at the time $t, \otimes$ is the Hamilton product, and $\delta t$ is the sampling time.

Computing the orientation quaternion from accelerometer and magnetometer values is less trivial. The idea of the algorithm, which is based on the Gauss-Newton method, is to exploit the information of gravity and Earth's magnetic flux obtained from the sensor to compute a measurement of rotation with respect to the Earth and limit the effects of drifting in the orientation estimate due to the gyroscope integration.

Let ${ }^{S} \mathbf{a}(t),{ }^{S} \mathbf{m}(t) \in \Re^{3 \times 1}$ be the accelerometer and magnetic components expressed in the sensor reference frame, and consider

$$
{ }^{S} \mathbf{e}(t)=\left[\begin{array}{c}
{ }^{S} \mathbf{a}(t) \\
{ }^{S} \mathbf{m}(t)
\end{array}\right] \in \Re^{6 \times 1} .
$$

We can refer to the known Earth reference vector as $W \mathbf{e}(t) \in \Re^{6 \times 1}$, and consequently we can define the orientation estimation error as

$$
\epsilon(t)={ }^{W} \mathbf{e}(t)-{ }^{W} \mathbf{M}_{S}(t){ }^{S} \mathbf{e}(t)
$$

The idea is to use the Gauss-Newton (GN) method to estimate ${ }^{W} \mathbf{R}_{S}(t)$ (and consequently ${ }^{W} \mathbf{M}_{S}(t)$ ) in Eq. (3). Let $\mathbf{r}(t)$ be the quaternion representation of the rotation matrix ${ }^{W} \mathbf{M}_{S}(t)$. If we write a single step of the GN method in a quaternion form we obtain

$$
\mathbf{r}_{i+1}(t)=\mathbf{r}_{i}(t)-\mathbf{J}_{i}^{\sharp}(t) \epsilon(t)
$$

where

$$
\mathbf{J}_{i}^{\sharp}(t)=\left(\mathbf{J}_{i}^{T}(t) \mathbf{J}_{i}(t)\right)^{-1} \mathbf{J}_{i}^{T}(t),
$$

the subscript $i$ represents the $i$-th iteration of the optimization algorithm and $\mathbf{J}_{i}(t)$ is the Jacobian of the error $\epsilon(t)$ introduced in Eq. (3). As suggested in [21], [22], we compensate the possible magnetic distortion forwarding the latest computed quaternion.

The last step of the algorithm fuses the estimation computed exploiting the accelerometer and magnetometer (see Eq. (4)) with the quaternion estimated with the gyroscope integration. This operation is provided by a complementary filter. The gyroscope orientation $\mathbf{g}(t)$ is fused with the GaussNewton method result $\mathbf{r}(t)$. The output quaternion $\mathbf{q}(t)$ is computed as,

$$
\mathbf{q}(t)=\gamma \mathbf{g}(t)+(1-\gamma) \mathbf{r}(t)
$$

being $0<\gamma<1, \gamma \in \Re$ the parameter of the complementary filter and

$$
\mathbf{g}(t)=\mathbf{q}(t-\delta t)+\dot{\mathbf{g}}(t) \delta t
$$

where $\dot{\mathbf{g}}(t)$ is in accordance with Eq. (2). For the sake of simplicity $\mathbf{g}(t)$ is initialized as

$$
\mathbf{g}(0)=\left[\begin{array}{llll}
1 & 0 & 0 & 0
\end{array}\right]^{T} \text {. }
$$

Please refer to [21] for more information about the performance of the proposed algorithm.

Let ${ }^{W} \mathbf{q}_{H}(t)$ be the quaternion that define the orientation of the frame associated to the head, and ${ }^{W} \mathbf{q}_{0}$ be the offset quaternion estimated during the initialization, both with respect to the global reference frame $W$. Thus, the orientation of the cap with respect to the new reference position is computed as

$$
{ }^{0} \mathbf{q}_{H}(t)={ }^{0} \mathbf{q}_{W}(t) \otimes{ }^{W} \mathbf{q}_{H}(t) .
$$

being ${ }^{0} \mathbf{q}_{W}(t)$ the conjugate quaternion of ${ }^{W} \mathbf{q}_{0}(t)$.

The cap orientation described by the quaternion ${ }^{0} \mathbf{q}_{H}(t)$ can be converted into Euler angles representation and therefore the tilt estimation results straightforward. The reference system and the rotations with respect to the initial orientation are shown in Fig. 4. These values are processed by the controller to actuate the robot, as explained in Section II

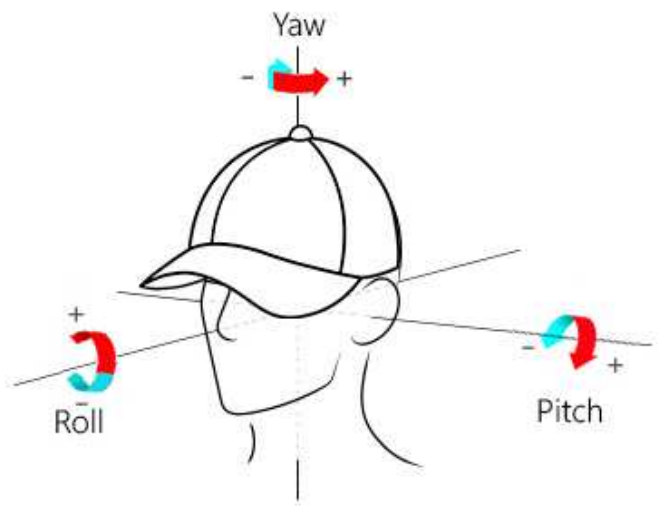

Fig. 4. Cap reference frame. We decide to use only Roll and Pitch rotation since the Yaw rotation is not desirable for people with impairments.

\section{EMG SIGNAL DETECTION}

Assistive devices aim at reproducing natural motion of the human arm, and usually can be controlled in three different modes: translation of the end effector along the three axis of the space, wrist rotation, and fingers opening/closing. To swap between one modality to another, we exploit the EMG interface substituting buttons and switches. Following the approach described in [23] we focused on the acquisition of the EMG signal generated when the user contracts the frontalis muscle. Thanks to its bilateral representation in the brain cortex the frontalis muscle is always spare, also in case of stroke, becoming a suitable candidate for substituting the aforementioned button functions [24]. An EMG interface measures the electrical potential between a ground and a signal electrode. The EMG signal can be measured either invasively, with wire or needle electrodes, or noninvasively with surface electrodes. The former consists of 

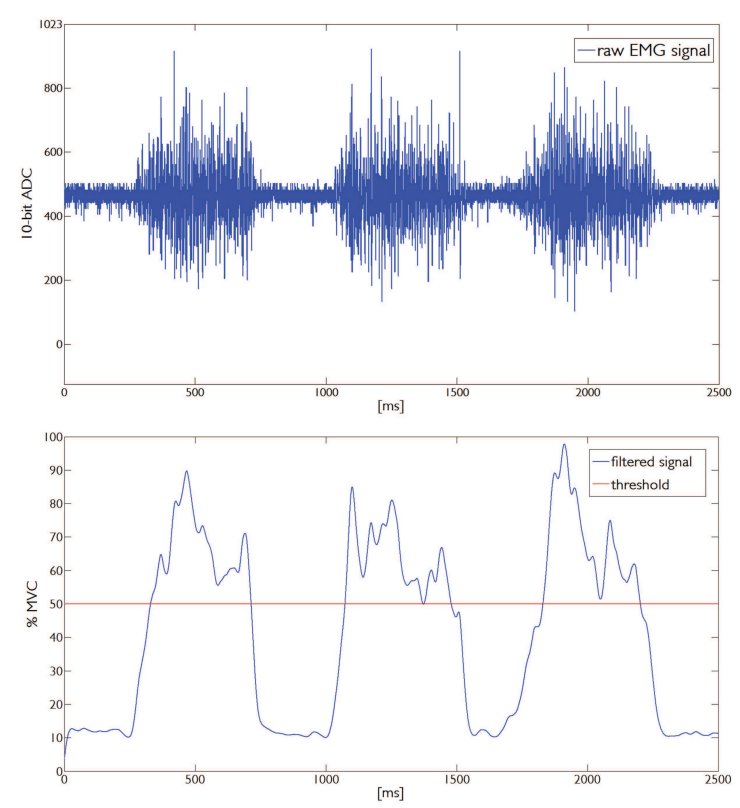

Fig. 5. Bottom: Example of three activations $\left(\right.$ event $e_{3}$ ) in a time window of 2,5 seconds. Top: raw EMG signal. Bottom: processed EMG signal after the operations of rectification, normalization and filtering (blue); threshold (red).

a wire electrode that is inserted into the muscle, whereas the latter consist in electrodes placed on the intact skin surface over the subjects' muscle. In the proposed approach we used the second method, i.e., surface EMG, in particular we used non-gelled reusable silver/silver-chloride surface electrodes. They present the lowest noise interface and are recommended for biopotentials recording [25]. For acquiring the signals, we designed and developed a custom acquisition board, fulfilling requirements on bandwidth, dynamic range, and physiological principles. A typical EMG waveform is characterized by a spectral content between 10 to $250 \mathrm{~Hz}$ with an amplitude up to $5 \mathrm{mV}$ depending on the particular muscle [26]. The microcontroller samples the EMG signal at $1 \mathrm{kHz}$ to avoid aliasing. The reference value of received EMG is normalized using the maximum voluntary contraction (MVC) technique [20]. Fig. 5 shows a representative raw EMG signal and its filtered values. The trigger signal is obtained using a single-threshold value defined as the $50 \%$ of the MVC, a level that was repeatable and sustainable for the subject without producing undue fatigue during the use of the interface. More in detail, to prevent false activations due to glitches or to spontaneous spikes, a trigger is detected only if the captured signal exceeds the threshold for at least $50 \mathrm{~ms}$ [27]. Moreover to generate multiple-contractions event we set a time window of 2,5 seconds starting from the first threshold overtaking. Thus, events $e_{1}, e_{2}, e_{3}$ correspond to one, two or three muscle contractions in the same time window, respectively.

\section{EXPERIMENTAL SETUP}

In this section, we present the experimental evaluation performed to assess the feasibility and functionality of our

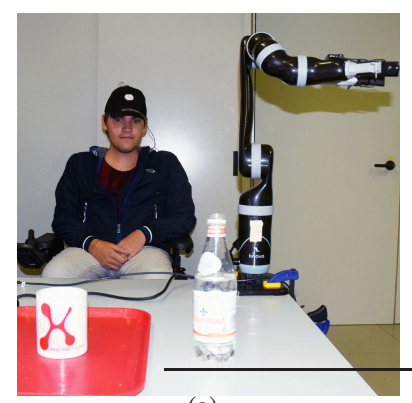

(a)

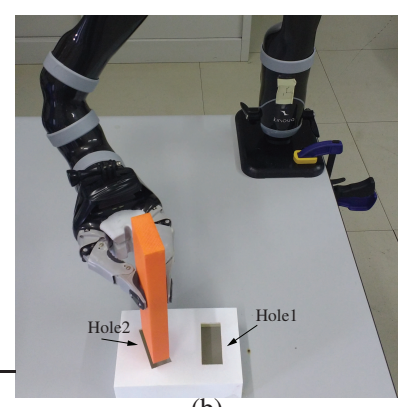

(b)
Fig. 6. Experimental setup. The user with his own wheelchair. He wears the hat and is able to control the robotic arm tilting the head. For the sake of simplicity in the experimental phase the robot was fixed to a table.

approach. We validated the proposed system in two different scenarios. In the first scenario, users were asked to fill a glass with a bottle of water. In this scenario, we tested the usability of the interface for ADL and its capacity in performing tasks with multiple translations and wrist rotations. We defined this scenario as $\mathrm{S} 1$. The second scenario is a pick and place task. This choice was adopted to highlight the capability of the system in performing fine rotations and translation. We refer to this scenario as S2. Users repeated the experiments in two different modalities. In the first modality (I), users wore the hat and controlled the robot with the proposed interface. In the second modality $(J)$ users used 2 axis and 6 buttons joystick to control the robot. The MICO2 robotic manipulator was mounted on a table, close to the left side of the wheelchair to provide users with disabilities more capabilities to interact and manipulate the objects in the environment. Ten subjects (age range 24-51, eight males) were involved in these tests, eight of them were healthy subjects while one of the participants reported serious limited mobility both to the upper and lower limbs, and another one had limited lower limbs mobility. The participants signed informed consent forms. All of them were informed about the purpose of the experiment, were able to discontinue participation at any time, and no payment was offered for the participation. A familiarization period of 5 minutes for each modality was provided to participants to acquaint them with the system. In the first scenario (S1), each user wore the cap and performed the proposed task. A representative user wearing the system and performing $\mathrm{S} 1$ is shown in Fig. 6(a). In S2 users were asked to pick a peg from a hole in the support base and place it inside another one with a different orientation (see Fig. 6(b)). The peg was a 3D-printed parallelepiped with a $2 \times 5 \mathrm{~cm}$ base and 20 $\mathrm{cm}$ height. The size of the holes in the support base was $2.5 \times 5.5 \mathrm{~cm}$, while the board was $3.5 \mathrm{~cm}$ high. The final hole (hole2) was rotated of 20 degrees with respect to starting hole (hole1) about the direction perpendicular to the punctured board surface.

In both scenarios, users tried the two modalities four times, an exception was made for the upper limb impaired user that performed only the $I$ modality. The $J$ modality was used as a 


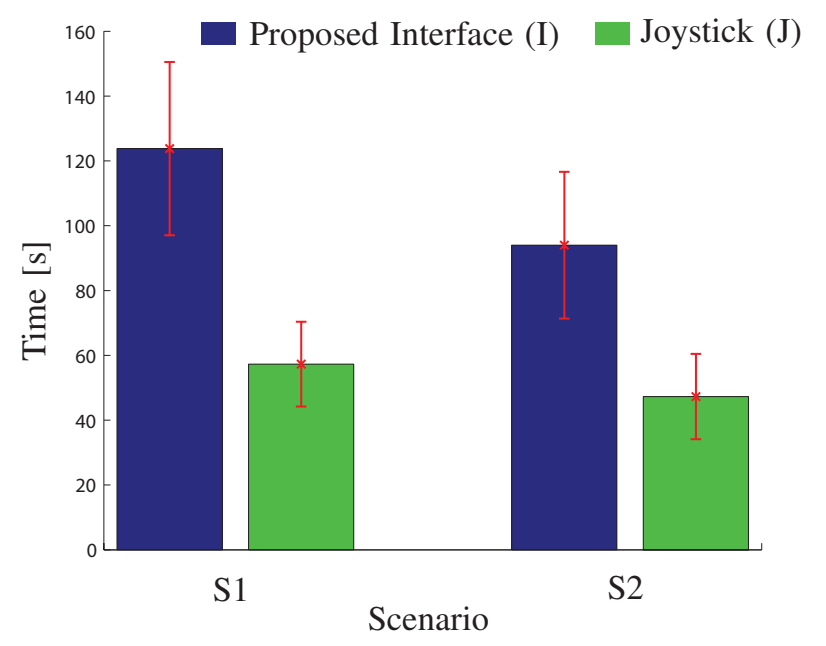

Fig. 7. Results of the experimental validation, divided by scenario: we report mean time ( \pm standard deviation) to fill a glass of water and to complete the pick and place task for S1 and S2, respectively. Blue bars represent data where users used the proposed interface $(I)$, whereas green bars represent data where users controlled the robot with the joystick $(J)$.

gold metric for evaluating the functionality of our approach. The average task completion times are summarized in Fig 7. Results shows the effectiveness of the proposed approach. An additional comparison metric can be found in [11], where the authors evalueted their interface on a task similar to $\mathrm{S} 1$; results of [11] undertook more than 200 seconds. At the end of the trials, an online survey based the Usability and User Experience (USE) [28] in the form of a bipolar Likerttype was proposed to the subjects. The USE questionnaire evaluates four dimensions of usability: usefulness, ease of use, ease of learning, and satisfaction. Each dimension is assessed with a number of items which subjects respond to with a seven-point scale $(1=$ strongly disagree, $7=$ strongly agree) for the overall system. Results are shown in Table II.

TABLE II

QUESTIONNAIRE FACTORS AND RELATIVE MARKS.

\begin{tabular}{|l|c|}
\hline Questionnaire factors & Mean (SD) \\
\hline \hline Usefulness & $5.9(0.3)$ \\
\hline Ease of use & $5.5(0.5)$ \\
\hline Ease of learning & $5.6(0.6)$ \\
\hline Satisfaction & $6.1(0.3)$ \\
\hline
\end{tabular}

Mark ranges from " $1=$ strongly disagree" to " $7=$ strongly agree". Mean and standard deviation (Mean (SD)) are reported.

From Table II, we can assert that the subjects rated positively the system. In particular the satisfaction after the trials results very high. On the other hand, the easiness of the interface use and learning is the weakest point. This is probably due to the limited familiarization time before the trials. The high variance is symptomatic of different perceptions between the users depending on their motor abilities.

\section{CONCLUSION AND FUTURE WORK}

Results of the users evaluation and time comparison, confirm the feasibility and greater usability of the proposed interface (see Table II) that implements in a wearable way a MEMS tilt estimation and an EMG signal detection to control a 6 DoF lightweight arm. A cap, instrumented with a nine axis MARG and three EMG sensors, is used both to move and control the opening/closing of the end-effector. The results of the user study show that individuals with severe motor impairments can operate assistive robotic manipulators using the proposed system interface. Moreover patients confirmed its ease of use and performances comparable with a joystick based controller (cf. Fig 7). In future work we will design a more compact electronic system, and a force feedback techniques will be evaluated to improve control performance.

\section{REFERENCES}

[1] Eurostat, "Disability statistics - barriers to social integration," 2012. [Online]. Available: http://ec.europa.eu/eurostat/statistics-explained/ index.php/Disability_statistics_-_barriers_to_social_integration\# Difficulties_with_basic_activities_and_personal_and_household_care_ activities

[2] C. Chung and R. Cooper, "Literature review of wheelchair-mounted robotic manipulation: user interface and end-user evaluation," in RESNA Annual Conference, 2012.

[3] D.-J. Kim, R. Hazlett-Knudsen, H. Culver-Godfrey, G. Rucks, T. Cunningham, D. Portee, J. Bricout, Z. Wang, and A. Behal, "How autonomy impacts performance and satisfaction: Results from a study with spinal cord injured subjects using an assistive robot," IEEE Transactions on Systems, Man, and Cybernetics-Part A: Systems and Humans, vol. 42, no. 1, pp. 2-14, 2012.

[4] G. Verburg, H. Kwee, A. Wisaksana, A. Cheetham, and J. van Woerden, "Manus: The evolution of an assistive technology," Technology and Disability, vol. 5, no. 2, pp. 217-228, 1996.

[5] V. Maheu, P. S. Archambault, J. Frappier, and F. Routhier, "Evaluation of the JACO robotic arm: Clinico-economic study for powered wheelchair users with upper-extremity disabilities," in Rehabilitation Robotics (ICORR), 2011 IEEE International Conference on. IEEE, 2011, pp. 1-5.

[6] H. Eftring and K. Boschian, "Technical results from manus user trials," in ICORR'99: International Conference on Rehabilitation Robotics, 1999.

[7] H. Fang, Z. Xie, and H. Liu, "An exoskeleton master hand for controlling DLR/HIT hand," in Proc. IEEE/RSJ International Conference on Intelligent Robots and Systems (IROS), 2009, pp. 3703-3708.

[8] M. Palankar, K. J. De Laurentis, R. Alqasemi, E. Veras, R. Dubey, Y. Arbel, and E. Donchin, "Control of a 9-dof wheelchair-mounted robotic arm system using a p300 brain computer interface: Initial experiments," in Robotics and Biomimetics, 2008. ROBIO 2008. IEEE International Conference on. IEEE, 2009, pp. 348-353.

[9] K. M. Tsui and H. A. Yanco, "Simplifying wheelchair mounted robotic arm control with a visual interface." in AAAI Spring Symposium: Multidisciplinary Collaboration for Socially Assistive Robotics, 2007, pp. 97-102.

[10] M. Nishimori, T. Saitoh, and R. Konishi, "Voice controlled intelligent wheelchair," in SICE, 2007 annual conference. IEEE, 2007, pp. 336340.

[11] H. Jiang, J. P. Wachs, and B. S. Duerstock, "Integrated vision-based robotic arm interface for operators with upper limb mobility impairments," in Rehabilitation Robotics (ICORR), 2013 IEEE International Conference on. IEEE, 2013, pp. 1-6.

[12] R. Vaidyanathan, B. Chung, L. Gupta, H. Kook, S. Kota, and J. D. West, "Tongue-movement communication and control concept for hands-free human-machine interfaces," IEEE Transactions on Systems, Man, and Cybernetics-Part A: Systems and Humans, vol. 37 no. 4, pp. 533-546, 2007. 
[13] R. Barea, L. Boquete, M. Mazo, and E. López, "System for assisted mobility using eye movements based on electrooculography," IEEE transactions on neural systems and rehabilitation engineering, vol. 10, no. 4, pp. 209-218, 2002.

[14] O. Fukuda, T. Tsuji, and M. Kaneko, "An EMG controlled robotic manipulator using neural networks," in Robot and Huтаn Cоттиnication, 1997. RO-MAN'97. Proceedings., 6th IEEE International Workshop on. IEEE, 1997, pp. 442-447.

[15] I. Oikonomidis, N. Kyriazis, and A. A. Argyros, "Efficient modelbased 3D tracking of hand articulations using Kinect." in BmVC, vol. 1, 2011, p. 3.

[16] C. Qian, X. Sun, Y. Wei, X. Tang, and J. Sun, "Realtime and robust hand tracking from depth," in Proc. IEEE Conference on Computer Vision and Pattern Recognition, 2014, pp. 1106-1113.

[17] D. Roetenberg, H. Luinge, and P. Slycke, "Xsens MVN: full 6DOF human motion tracking using miniature inertial sensors," Xsens Motion Technologies BV, Tech. Rep, 2009.

[18] E. M. Foxlin, "Head tracking relative to a moving vehicle or simulator platform using differential inertial sensors," in AeroSense 2000 International Society for Optics and Photonics, 2000, pp. 133-144.

[19] T. Lisini Baldi, M. Mohammadi, S. Scheggi, and D. Prattichizzo, "Using inertial and magnetic sensors for hand tracking and rendering in wearable haptics," in Proc. IEEE World Haptics Conference (WHC), 2015, pp. 381-387.

[20] D. Farina and R. Merletti, "Comparison of algorithms for estimation of emg variables during voluntary isometric contractions," Journal of Electromyography and Kinesiology, vol. 10, no. 5, pp. 337-349, 2000.

[21] D. Comotti, "Orientation estimation based on gauss-newton method and implementation of a quaternion complementary filter," 2011.

[22] S. Madgwick, A. Harrison, and R. Vaidyanathan, "Estimation of imu and marg orientation using a gradient descent algorithm," in Proc. IEEE International Conference on Rehabilitation Robotics, 2011, pp. $1-7$.

[23] I. Hussain, G. Salvietti, G. Spagnoletti, and D. Prattichizzo, "The SoftSixthFinger: a Wearable EMG Controlled Robotic Extra-Finger for Grasp Compensation in Chronic Stroke Patients," IEEE Robotics and Automation Letters, vol. 1, no. 2, pp. 1000-1006, 2016.

[24] A. Brodal, Neurological anatomy in relation to clinical medicine. Oxford University Press, USA, 1981.

[25] R. Merletti, A. Botter, A. Troiano, E. Merlo, and M. A. Minetto, "Technology and instrumentation for detection and conditioning of the surface electromyographic signal: state of the art," Clinical Biomechanics, vol. 24, no. 2, pp. 122-134, 2009.

[26] A. Merlo and I. Campanini, "Technical aspects of surface electromyography for clinicians," The Open Rehabilitation Journal, vol. 3, no. 1, 2010.

[27] P. Konrad, "The abc of emg," A practical introduction to kinesiological electromyography, vol. 1, pp. 30-35, 2005.

[28] A. M. Lund, "Measuring Usability with the USE Questionnaire," 2001. 